\title{
Ameloblastoma of the mandible in a male dog - A Case Report
}

\author{
Shivakumar B. $\mathrm{N}^{1}$ and Manohara Upadhya* \\ Mangalore Veterinary Service Centre, Manglore, Karnataka, India. \\ 1. Institute of Dental Sciences, Ghatikia, Bhuvaneshwar, Orissa, India. \\ * Corresponding author email : mantapa@gmail.com \\ Published Online : 30-04-2011
}

\begin{abstract}
Ameloblastomas are odontogenic tumors known for their aggressive nature. Because of the odontogenic origin, they are seen affecting the mandible and maxilla. Like humans, animals like dogs, horses may also develop these lesions. In this report, ameloblastoma of the mandible in a male dog is described, The lesion clinically appeared like an exophytic growth on the gingiva near mandibular left canine tooth, radiograph showed a large multilocular radioleucency with destruction of the lower mandible. The lesion recurred after 2 years following the conservative removal. This case report is interesting because similar to humans, the dogs too suffer from the odontogenic lesions. The radiographic, histologic features and the behaviour pattern were similar to humans' where as the treatment rendered was only conservative keeping the limited life span of the dog.
\end{abstract}

Keywords: Ameloblastoma, Canine, Mandible, Odontogenic tumor.

\section{Introduction}

Ameloblastomas (Adamantinomas) are tumours arising from the tooth forming structures. These tumors, though benign, may be locally destructive. Since the tumors develop from odontogenic tissues, they arise from the alveolar border of maxilla or mandible, though clinically appear to arise from the gums [1].

Historically, this lesion has been known for many years, with reports dating back to the nineteenth century [2]. Ameloblastomas are frequent in animals. Though cattle are more commonly affected, the tumour has been seen in dogs, horses, mules and cats [1]. There are few case reports on the ameloblastomas occurring in dogs [3].

\section{Case Description}

A 10 year old male dog was brought to the veterinary clinic at Mangalore, with the complaint of a growth on the gingiva protruding out in the anterior region of the mouth. On examination an exophytic growth measuring $2 \mathrm{X} 3 \mathrm{cms}$. was seen extending from the gums adjacent to left canine tooth (Fig.1).

Consistency of the mass was firm and it appeared firmly attached to the gingival tissues. Radiograph of the mandible showed a large destructive radiolucent lesion, displacing the root, also causing thinning of the cortical plate and discontinuity of the lower border of the mandible in that region (Fig.2). Since the dog was unable to close the mouth and it was finding difficulty in eating, it was decided to excise the mass. The excised specimen histologically consisted of mass of odontogenic tissues. There were follicles, strands made up of ameloblast like cells in the periphery and loosely arranged stellate shaped cells in the centre. ( Fig.3) . The tumour mass had fused with the surface epithelium. The case was diagnosed as Plexiform Ameloblastoma with follicular areas.

\section{Discussion}

Robinson defined Ameloblastoma as a benign tumour that is "usually unicentric, nonfunctional, intermittent in growth, anatomically benign and clinically persistent"'[4]. This odontogenic tumour that frequently occurs intraosseously perforates the alveolar ridge and fuses with the oral cavity. This tumour since locally aggressive and grows rapidly, needs a radical approach. Also, the recurrence rate is high after conservative treatment. In the present case, the first surgical treatment of local excision with cauterization resulted in temporary relief.

Though the tumour has recurred after 2 years, it slowly enlarged and became symptomatic, after 6 months. Since the radical approach involves resection of a part of the mandible, it was decided to treat conservatively once again, as the dog was old and radical approach might result in functional impairment in the absence of prosthesis. The conservative treatment was surgical excision of clinically visible lesion with cauterization. 




Figure-1. Clinical photograph showing the gingival growth on the left anterior gingiva of the dog.

\section{Conclusion}

To conclude. This presentation is about a case of Ameloblastoma of the mandible in a dog, where the tumour has perforated into the oral cavity leading to a growth on the gingiva, with very little expansion of the bone. Here, the conservative treatment given resulted in the recurrence of the tumour after 2 years, where once again it was treated conservatively, considering the old age of the dog.

Clinical Significance: The odontogenic lesions occur in animals too. The detailed study and documentation

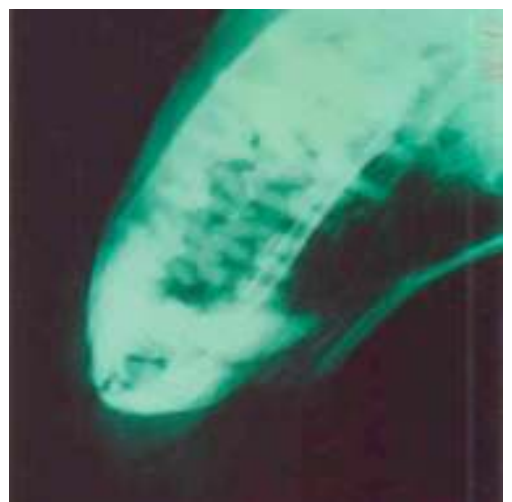

Figure-2. Picture of the radiograph showing the large destructive, radiolucent lesion.



Figure-3. Photomicrograph of the lesion showing the odontogenic epithelial cells arranged in follicles; periphery shows ameloblast like cells, the centre shows stellate reticulum like cells. (H\&E staining, 10X magnification)

of such lesions help in understanding the biological behavior and can act as study models.

\section{References}

1. Regezi and Sciubba (1993) Oral Pathology: ClinicalPathologic correlations, W.B saunders, $2^{\text {nd }}$ edition; pages-363.

2. Sakai H, et.al. (1996). Ameloblastoma with prominent ossification in the mandible of a dog. J Vet Med Sci. Nov;58(11);1113-5

3. Sastry Ganti A (1983) Veterinary Pathology, CBS Publishers $\&$ Distributors, $6^{\text {th }}$ edition; pages- 265 .

4.. Shafer WG, Hine MK, Levy BM (1993). A Text book of Oral Pathology, W.B.Saunders, $4^{\text {th }}$ edition; pages- 276. 